an additive effect of the two agents when administered together. These results will be presented. In addition, results from on-going PKPD studies of TMZ in combination with two other small molecule inhibitors, RG7388, an MDM2 inhibitor, and GDC0068, an AKT inhibitor, will also be presented. DISCUSSION/SIGNIFICANCE OF IMPACT: Our long-term goals are to further elucidate SOC-induced responses in GBM and establish combination treatment regimens that are safe and significantly improve therapeutic efficacy. Collectively, our studies will broadly influence chemotherapy of GBM by establishing a process to rationally design combination approaches that mitigate resistance development. These studies will ultimately provide opportunities to study other targeted agents tailored to individual molecular signatures of GBM, as well as other tumor types.

3145

\section{An Evaluation of Machine Learning and Traditional Statistical Methods for Discovery in Large-Scale Translational Data \\ Megan C Hollister ${ }^{1}$ and Jeffrey D. Blume ${ }^{1}$ \\ ${ }^{1}$ Vanderbilt University Medical Center}

OBJECTIVES/SPECIFIC AIMS: To examine and compare the claims in Bzdok, Altman, and Brzywinski under a broader set of conditions by using unbiased methods of comparison. To explore how to accurately use various machine learning and traditional statistical methods in large-scale translational research by estimating their accuracy statistics. Then we will identify the methods with the best performance characteristics. METHODS/STUDY POPULATION: We conducted a simulation study with a microarray of gene expression data. We maintained the original structure proposed by Bzdok, Altman, and Brzywinski. The structure for gene expression data includes a total of 40 genes from 20 people, in which 10 people are phenotype positive and 10 are phenotype negative. In order to find a statistical difference $25 \%$ of the genes were set to be dysregulated across phenotype. This dysregulation forced the positive and negative phenotypes to have different mean population expressions. Additional variance was included to simulate genetic variation across the population. We also allowed for within person correlation across genes, which was not done in the original simulations. The following methods were used to determine the number of dysregulated genes in simulated data set: unadjusted p-values, BenjaminiHochberg adjusted p-values, Bonferroni adjusted p-values, random forest importance levels, neural net prediction weights, and secondgeneration p-values. RESULTS/ANTICIPATED RESULTS: Results vary depending on whether a pre-specified significance level is used or the top 10 ranked values are taken. When all methods are given the same prior information of 10 dysregulated genes, the BenjaminiHochberg adjusted p-values and the second-generation p-values generally outperform all other methods. We were not able to reproduce or validate the finding that random forest importance levels via a machine learning algorithm outperform classical methods. Almost uniformly, the machine learning methods did not yield improved accuracy statistics and they depend heavily on the a priori chosen number of dysregulated genes. DISCUSSION/SIGNIFICANCE OF IMPACT: In this context, machine learning methods do not outperform standard methods. Because of this and their additional complexity, machine learning approaches would not be preferable. Of all the approaches the second-generation p-value appears to offer significant benefit for the cost of a priori defining a region of trivially null effect sizes. The choice of an analysis method for largescale translational data is critical to the success of any statistical investigation, and our simulations clearly highlight the various tradeoffs among the available methods.

\section{An Injectable Sulfonated Reversible Thermal Gel for Controlled and Localized Delivery of Vascular Endothelial Growth Factor to Promote Cardiac Protection After a Myocardial Infarction}

Adam J Rocker ${ }^{1}$, David Lee ${ }^{1}$, Maria Cavasin ${ }^{1}$ and Daewon Park ${ }^{1}$

${ }^{1}$ University of Colorado Denver

OBJECTIVES/SPECIFIC AIMS: This study aims to evaluate an injectable sulfonated reserve thermal gel (SPSHU-PNIPAM) for angiogenic growth factor delivery by examining the vascularization and cardioprotective properties of the polymer system. This study could lead to clinical translation by moving into larger animal studies and eventually clinical trials. The success of this study was determined by analyzing the results of echocardiography data on cardiac function (ejection fraction, fractional shortening, and left ventricle inner diameter) and assessment of histological staining on cardiac tissue (fibrotic tissue formation, infarct size, wall thinning, blood vessel cell counts, and vessel size quantification) after MI. Five groups were compared for this study: saline, VEGF, SPSHU-PNIPAM, SPSHU-PNIPAM loaded with VEGF, and no injection (sham). Significant statistical differences between control groups and polymer injection groups, when $\mathrm{p}<0.05$, indicates successful outcomes from this study. METHODS/STUDY POPULATION: SPSHUPNIPAM Polymer Synthesis: SPSHU-PNIPAM was synthesized as previously described. Briefly, PSHU was synthesized with N-BOC serinol, urea, and HDI at $90{ }^{\circ} \mathrm{C}$ for 7 days. PSHU was deprotected in DCM and TFA at room temperature for $45 \mathrm{~min}$. PNIPAM was conjugated to the deprotected PSHU using EDC and NHS at room temperature for $24 \mathrm{~h}$. PSHU-PNIPAM was sulfonated with 1,3-propanesultone and potassium tert-butoxide at $60^{\circ} \mathrm{C}$ for 3 days. Surgical Procedure: Male C57BL/6 mice weighing 24-28 g were anaesthetized using isoflurane and artificial ventilation provided. A small left thoracotomy incision was made at the left fourth intercostal space to expose the heart, and the proximal left anterior descending coronary artery was ligated for $45 \mathrm{~min}$. The coronary artery was then released and $30 \mu \mathrm{l}$ injections of saline, SPSHUPNIPAM (1\% w/v), bolus VEGF (200 ng), or SPSHU-PNIPAM + VEGF $(1 \%, 200 \mathrm{ng})$ were injected intramyocardially at the infarcted site and the incision closed. Echocardiography and Histological Staining: Standard serial transthoracic echocardiography was performed while simultaneously recording ECG to assess cardiac morphology and left ventricular function. Immunohistochemistry and histology staining procedures were used to identify: fibrotic tissue formation, infarct size, wall thinning, blood vessel cell counts, and vessel size quantification. These were performed according to manufacturer instructions or by previously published criteria. Statistical Analysis: Two-tailed t-test assuming unequal variances was used to determine significant differences between two groups. Analysis of variance (ANOVA) was used to determine significant differences between three or more groups followed by Tukey-Kramer to determine significant differences between two groups as appropriate. Statistical significance was considered when $\mathrm{p}<0.05$. References: Lee, D. J., Rocker, A. J., Bardill, J. R., Shandas, R. and Park, D. (2018), A sulfonated reversible thermal gel for the spatiotemporal control of VEGF delivery to promote therapeutic angiogenesis. J Biomed Mater Res. doi:10.1002/jbm.a.36496. RESULTS/ANTICIPATED RESULTS: Echocardiography results: Ejection fraction improved for 
the SPSHU-PNIPAM groups compared to the saline, VEGF, and no injection controls (Figure 1). SPSHU-PNIPAM either loaded with or without VEGF seemed to have very similar treatment effects for ejection fraction and fractional shortening. This indicates that the more significant component of the cardioprotective effects of the hydrogel system is the biomaterial itself rather than the release of VEGF (Figure 1). However, the only statistically significant improvement for ejection fraction, fractional shortening, and left ventricular inner diameter that was observed compared to the saline, VEGF, and no injection controls was the SPSHU-PNIPAM + VEGF group (Figure 1). Histology Results: After analyzing Masson trichrome staining, SPSHU-PNIPAM + VEGF demonstrated the smallest infarct size after MI reperfusion injury and was statistically reduced compared to the saline, VEGF, and no injection controls (Figure 2). Furthermore, left ventricular wall thickness showed that the SPSHUPNIPAM + VEGF treatment group reduced the wall thinning resulting from MI. The SPSHU-PNIPAM group without VEGF displayed a thicker ventricular wall as well, which may be attributed to the increased mechanical stability with the intramyocardial injection of the biomaterial (Figure 2). The immunohistochemical results for vascularization show that the SPSHU-PNIPAM + VEGF group significantly increased the number of functional vascular endothelial cells compared to the saline, VEGF, SPSHU-PNIPAM, and no injection controls (Figure 3). Additionally, the SPSHU-PNIPAM + VEGF group showed a significant increase in total vessel formation compared to the control groups, although there was no significant difference compared to SPSHU-PNIPAM without VEGF (Figure 3). The promotion of angiogenesis, without the delivery of VEGF, may be attributed to inflammation induced vascularization, including VEGF dependent vascularization that is initiated via signal transducer and activator of transcription 3 (STAT3) pathway that is induced by the pro-inflammatory cytokine interleukin 6. DISCUSSION/ SIGNIFICANCE OF IMPACT: The SPSHU-PNIPAM loaded with VEGF was evaluated for therapeutic angiogenesis to protect cardiac function after MI. Treatment with SPSHU-PNIPAM showed improved cardiac function and vascularization; however, the additional delivery of VEGF showed inadequate additional therapeutic benefits. Further investigation will include optimizing VEGF release characteristics including both loading amount and release rate. The decline of ejection fraction and fractional shortening after MI were reduced, while left ventricular internal diameter showed reduced ventricular dilation. Both infarct size and left ventricular wall thinning decreased while an increase in the vessel formation was observed. These results demonstrate the SPSHU-PNIPAM biomaterial has cardioprotective and increased vascularization properties for the treatment of MI.

3265

\author{
Analysis of High-Dimensional Patient Data in \\ Characterizing Alzheimer's Disease Progression \\ Daniel Baer ${ }^{1}$, Andrew B. Lawson ${ }^{1}$, Brandon Vaughan ${ }^{1}$ and \\ Jane E. Joseph ${ }^{1}$ \\ ${ }^{1}$ Medical University of South Carolina
}

OBJECTIVES/SPECIFIC AIMS: Our research hypothesis is that resting state fMRI (rsfMRI) data can be used to identify regions of the brain which are associated with cognitive decline in patients thereby providing a tool by which to characterize $\mathrm{AD}$ progression in patients. METHODS/STUDY POPULATION: We used data from the Alzheimer's Disease Neuroimaging Initiative (ADNI) to analyze
Mini-Mental State Examination (MMSE) questionnaire scores from 14 patients diagnosed with $\mathrm{AD}$ at two measurement occasions. RsfMRI data was available at the first of these occasions for these patients. These rsfMRI data were summarized into 264 node-based graph theory measures of clustering coefficient and eigenvector centrality. To address our research hypothesis, we modeled changes in patient MMSE scores over time as a function of these rsfMRI data, controlling for relevant confounding factors. This model accounted for the high-dimensionality of our predictor data, the longitudinal nature of the outcome, and our desire to identify a subset of regions in the brain most associated with the MMSE outcome. RESULTS/ ANTICIPATED RESULTS: The use of either the clustering coefficient or eigenvector centrality rsfMRI predictors in modeling MMSE scores for patients over time resulted in the identification of different subsets of brain regions associated with cognitive decline. This suggests that these predictors capture different information on patient propensity for cognitive decline. Further work is warranted to validate these results on a larger sample of ADNI patients. DISCUSSION/SIGNIFICANCE OF IMPACT: We conclude that different rsfMRI graph theory measures capture different aspects of cognitive function and decline in patients, which could be a future consideration in clinical practice.

3449

\section{Bacterial biotransformation of chemotherapeutics may} promote diversity among the intestinal microbiota

Ryan Andrew Blaustein ${ }^{1}$, Patrick Casey Seed ${ }^{1}$ and

Erica Marie Hartmann ${ }^{1}$

${ }^{1}$ Northwestern University

OBJECTIVES/SPECIFIC AIMS: This study aims to test the hypothesis that bacterial biotransformation of chemotherapeutics promotes gut microbial diversity by enhancing persistence of drug-sensitive taxa. METHODS/STUDY POPULATION: The impacts of doxorubicin on a model community of gut bacteria was investigated in vitro in anaerobic batch culture. The synthetic community was composed of specific members predicted by genomic analysis to be sensitive to the therapeutic (i.e., Clostridium innocuum, Lactobacillus sp.), resistant via putative biotransformation (i.e., Escherichia coli, Klebsiella pneumoniae), or resistant via putative efflux (i.e., Enterococcus faecalis). Bacterial growth was monitored in monocultures by measuring OD600 and standard plate counts, and in mixed cultures by strain-targeted qPCR. Doxorubicin concentration was detected via absorbance assay. RESULTS/ANTICIPATED RESULTS: Strains with predicted resistance to doxorubicin by drug biotransformation significantly lowered concentrations of the drug in culture media. In contrast, E. faecalis proved resistant without evidence of drug transformation. Predicted sensitive strains were growth-repressed by the doxorubicin, but able to grow in spent media where biotransformation had occurred. However, they remained growth-repressed in spent media from E. faecalis where drug transformation had not been observed. Bacterial growth kinetics in mixed batch culture were dependent on starting bacterial concentrations and timing of drug exposure. DISCUSSION/SIGNIFICANCE OF IMPACT: This work will be extended to model microbial community responses to doxorubicin as a factor of microbial interactions and extent of drug transformation prior its exposure to sensitive strains. The resulting model will have translational implications for mitigating health risks during pediatric cancer treatment. 